\title{
Influence of Interconnection Surface Finishes on Quality of Adhesive Joints
}

\author{
P. Mach, D. Bušek, A. Duraj \\ Department of Electrotechnology, Faculty of Electrical Engineering, Czech Technical University in Prague, \\ Prague, Czech Republic \\ mach@fel.cvut.cz
}

\begin{abstract}
Electrically conductive adhesives electrically connect and mechanically bond circuits to a variety of substrates. Four different silver-filled isotropically conductive adhesives have been used for attaching of 1206 chip components (resistors with "zero" values - jumpers) on printedcircuit boards with six different interconnection surface finishes: $\mathrm{Cu}$ (standard cleaning), oxidized $\mathrm{Cu}, \mathrm{Cu}$ after degradation vapors of $\mathrm{H}_{2} \mathrm{SO}_{3}, \mathrm{Ni}$, Au over $\mathrm{Ni}$, Sn (HAL). The resistances, nonlinearity, and noise of joints have been investigated before and after application of the dynamic mechanical load, which has been realized by periodical deflection of the printed circuit boards with assembled resistive chips. The load of the individual joints has been calculated. It has been found that degradation of $\mathrm{Cu}$ surface does not cause significant changes of electrical properties of the joints. The worst electrical parameters as well as the lowest resistance against the dynamic mechanical load have been found for the joints realized on Ni and Sn surface finishes.
\end{abstract}

\section{INTRODUCTION}

The use of electrically conductive adhesives for electronic packaging is joined with many different problems [1]. Two significant problems are:

- Does material of a pad influence quality of an adhesive joint significantly?

- Is surface finish of a pad significant for quality of the joint?

These problems have been solved elsewhere [2], [3]. However, the authors have evaluated electrical quality of the joints according to their resistances only. We have found [4] that noise and nonlinearity of the current vs. voltage characteristics of the joint describe its quality with substantially higher sensitivity, which makes the study with higher accuracy possible.

The second reason, why we have started this research work, has been impossibility to find properties of adhesive joints realized by attaching of chips on degraded $\mathrm{Cu}$ surfaces (e.g. by oxidation) in the literature. On the one hand, the producers have to avoid such the situation. On the other hand, it is not possible to exclude it, because the contacts are very often covered with thin layer of an oxide, which has higher or lower thickness.

Another reason, why we have directed our interest toward to this problem is, that it is possible to meet with requirement to mount components with different types of surface finish on one board. The question is which would be quality of the joints fabricated by adhesive, usually used for joining of finishes for adhesive joining, if the finishes would be of another type?

And, finally, it has been also interesting to investigate influence of the dynamic mechanical loading on properties of these joints.

\section{Conductivity of AdHesive JoInts}

Electrically conductive adhesive consists of binder and filler. Material of binder is usually epoxy or polyimide resin; as filler conductive particles of two shapes, balls and flakes, are used. These particles are usually of silver, but other metals such as gold, nickel, palladium or copper covered by silver film, have been tested, too. 
The electrical conductivity of isotropically conductive adhesive is uniform in all directions. Anisotropically conductive adhesive has different electrical conductivity in different directions. Adhesive joints fabricated of this type of adhesive have high conductivity in the direction perpendicular to the plane of the pad and very low conductivity in other directions. Therefore they are also named " $\mathrm{Z}$ adhesives." The work has been focused on investigation of properties of joints made of different types of isotropic adhesives on surfaces of different materials and with different finishes.

The total resistance of the adhesive joint consists of three parts:

- The resistance between adhesive and the pad

- The resistance of adhesive

- The resistance between adhesive and the lead of the component.

\subsection{The resistance between adhesive and the pad}

The resistance between adhesive and the pad depends on the type of the material of the pad and on the type and quality of its finish. If the surface of the pad would be oxidized, tunneling can take part in conductivity of such the contact if the thickness of the oxide layer will be sufficiently low. If the process of tunneling will be inelastic, then its current vs. voltage characteristics will be nonlinear. The resistance of a tunneling junction depends on the thickness of the tunneling barrier according to the formula

$$
R \approx \exp \left(d_{0} / D\right)
$$

Where $R \quad \ldots$ resistance of the tunneling junction, $d_{0} \ldots$ thickness of the insulating barrier, $D \ldots$ constant, $D \approx 0.1$ through $0.7 \mathrm{~nm}$.

It results from the equation (1) that a small increase of the thickness of the junction will cause high change of the resistance of the junction. Therefore tunneling between adhesive and the pad will cause nonlinearity of the current vs. voltage characteristics and, in dependence on the thickness of the tunneling barrier, increase of resistance of the joint.

Other possible mechanisms of the conductivity of a contact between a metal particle of filler and a pad covered by an oxide film are thermal emission, if the contact will be of a punctual type, and diffusion through the oxide barrier. Both these mechanisms are also nonlinear.

\subsection{The resistance of adhesive}

The resistance of adhesive depends on quality of a conductive net, which is created inside adhesive during its curing. The volume of adhesive decreases and quality of contacts among particles of filler improves. However, it is not possible to avoid, in general, also tunneling, thermal emission and diffusion among the particles. Quality of the conductive net depends on many parameters of adhesive: on diameter of conductive balls and magnitude of conductive flakes (if ball or flakes are used as filler), filling of resin with conductive particles, density of resin, and many others. Every producer of electrically conductive adhesives pays great effort to fabricate adhesive with the highest quality of conductive net. It is possible to assume that good adhesives will have resistivity comparable with the solders and that the adhesive joints made of such the adhesives will also have very low nonlinearity and noise

\subsection{The resistance between adhesive and the lead of the component}

It is possible to state that the analysis of possible mechanisms of conductivity, which has been carried out for the contact between adhesive and the pad, can be accepted also for the contact between adhesive and the lead of the component.

\subsection{Comparison of electrical properties of adhesive and soldered joints.}

Soldering is based on diffusion of atoms of the solder into the lattice of the soldered material. Solder is alloy, and therefore it is a material with minimum number of inhomogeneities.

Adhesive joining using electrically conductive adhesives creates joints of another type. The contact between particles of filler and joined material consists of a high number of punctual contacts and the same situation is inside the adhesive. Therefore as to the conductivity of the adhesive joint, it can be comparable with the conductivity of the soldered joint, but nonlinearity and noise of the adhesive joints will be higher. 


\section{EXPERIMENTAL}

The adhesive joints have been realized on following surfaces:

- Clean Cu pads.

- $\mathrm{Cu}$ pads degraded in $100 \%$ R.H. (the test boards have been located inside a wet desiccator for 14 days)

- $\mathrm{Cu}$ pads exposed in vapors of $\mathrm{H}_{2} \mathrm{SO}_{3}$. The mass of $\mathrm{H}_{2} \mathrm{SO}_{3}$, which has been needed for degradation, has been, for known volume of a desiccator and concentration of the acid, calculated according to the standard CSN EN 8130.

- $\mathrm{Cu}$ pads covered by thin Ni film. The film has been fabricated by galvanic plating; the thickness of the film has been $15 \mu \mathrm{m}$.

- $\mathrm{Cu}$ pads covered first by $\mathrm{Ni}$ film and then by galvanically plated Au film. The thickness of the Au film has been $5 \mu \mathrm{m}$.

- $\mathrm{Cu}$ pads covered by $\mathrm{Sn}$ film by hot air levelling (HAL)

Layout of the test board is shown in Fig. 1.

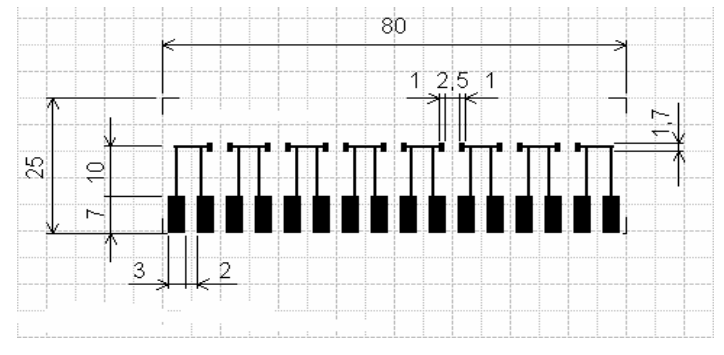

Fig. 1 Layout of the test board. The dimensions are in $\mathrm{mm}$. The basic material is FR4, thickness of $\mathrm{Cu}$ is $35 \mu \mathrm{m}$.

The adhesive joints have been fabricated by mounting of resistors of "zero" resistance on test boards by adhesives. Adhesives have been applied by dispensing. Following types of adhesives have been examined: SC70MN, ECO SOLDER AX70MN, ECO SOLDER AX20, and ELPOX ER55MN. All the adhesives have been delivered by the company Amepox Microelectronics.

Following parameters have been measured on adhesive contacts: resistance, nonlinearity of current vs. voltage characteristics and noise. The resistance has been measured in four- point arrangement using of an HP Precision RLC Meter
4284A. The measurement of nonlinearity has been described elsewhere [4]. The working place for the measurement of nonlinearity is shown in Fig. 2, the screen of the spectral analyzer during the measurement in Fig. 3.

The measurement of noise of the joints has also

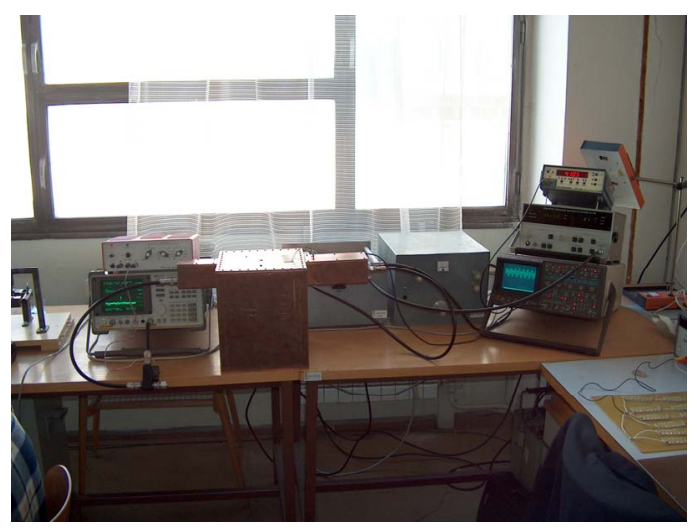

Fig. 2 The working place for the measurement of nonlinearity of the I-V characteristics of the adhesive joints.

The spectral analyzer HP 8560 is on the left, the power generator and filter in the middle, the oscilloscope and the second generator with the counter on the right.

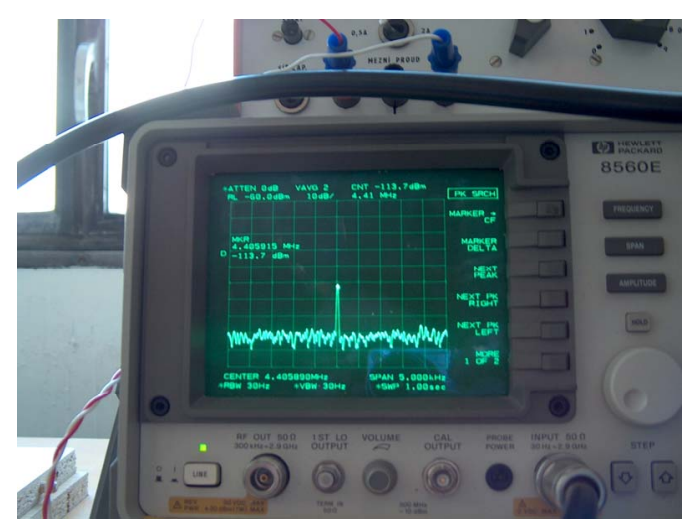

Fig. 3. Screen of the spectral analyzer during the measurement. The peak shows frequency and amplitude of the measured third harmonic.

been described elsewhere [4]. The working place for this measurement is shown in Fig. 4. 


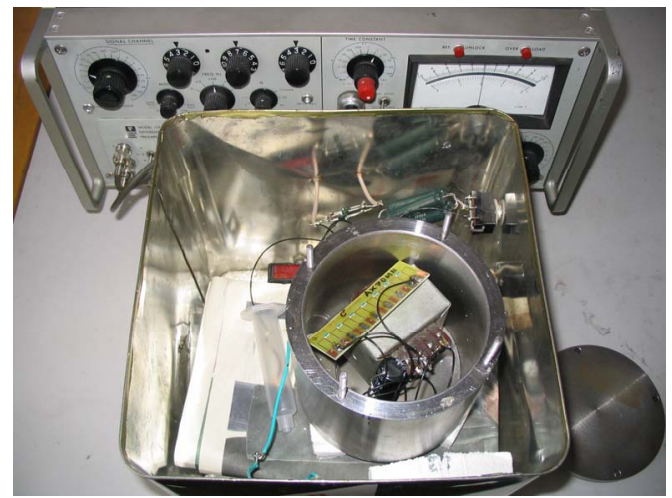

Fig. 4 The measurement of noise. Behind the screening box is the lock-in amplifier PAR $124 \mathrm{~A}$.

When the basic electrical characteristics of the joints have been measured, the joints have been loaded by the dynamic mechanical load. The test board has been horizontally fasted in a holder on one short side. Then it has been, on the opposite side, deflected by 3 $\mathrm{mm}$ up and down. The deflection of $\pm 3 \mathrm{~mm}$ has been chosen with respect to the calculated values of the tensile force and the shear force. The tensile force has been in the range of 0.3 through $2.5 \mathrm{~N}$ and the shear force in the range of 12 through $57 \mathrm{~N}$ (according to the position of the joint on the board). The number of deflections has been 1000 .

\section{RESUltS AND DISCUSSION}

The results of the measurements of the joints of adhesive SC 70MN are shown in Fig. 5, Fig. 6 and Fig. 7. The results measured on other adhesives under the test have been very similar as for the influence of surface finish on the electrical parameter, the absolute

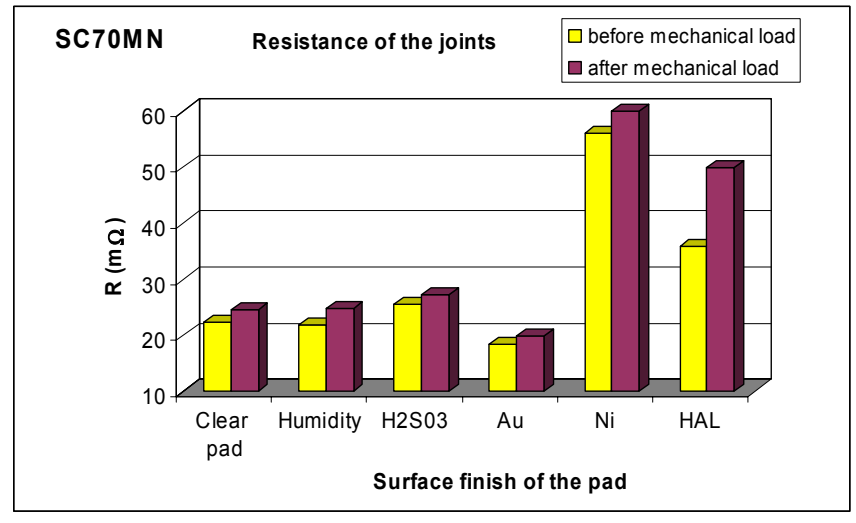

Fig. 5 Resistance of the joints of adhesive SC 70MN fabricated on different finishes of the pads before and after the mechanical load.

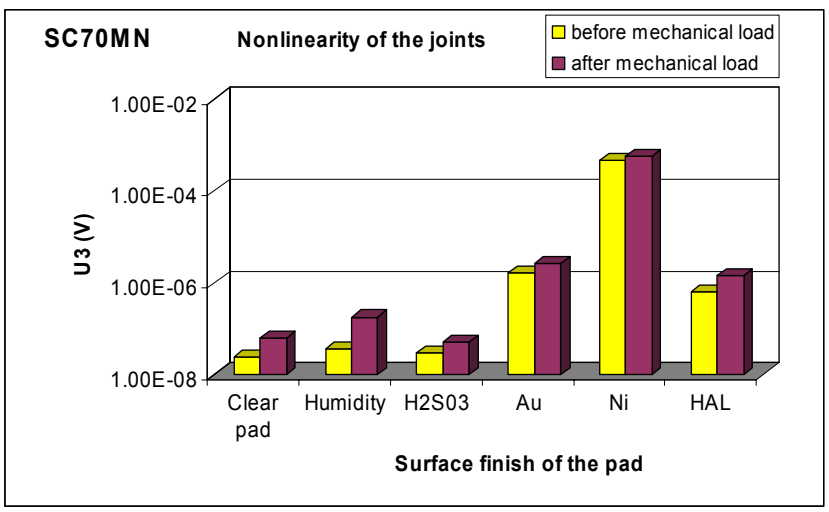

Fig. 6 Nonlinearity of the joints of adhesive SC 70MN fabricated on different finishes of the pads before and after the mechanical load.

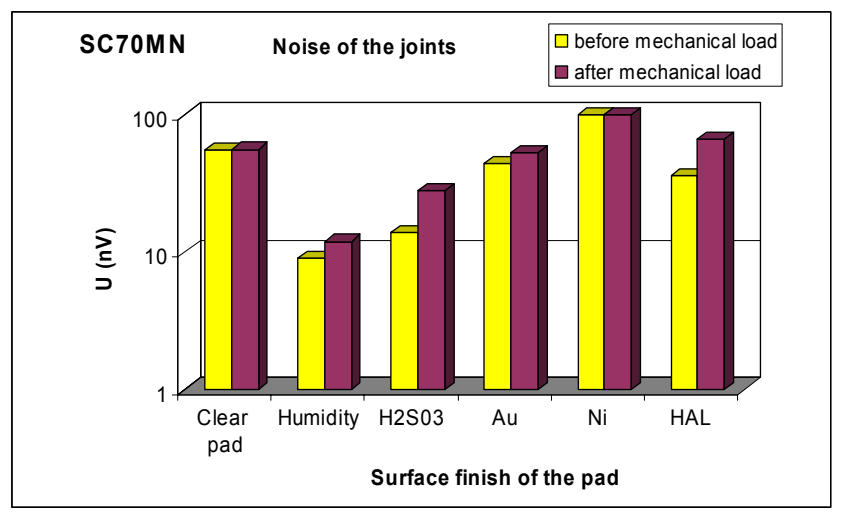

Fig. 5 Noise of the joints of adhesive SC 70MN fabricated on different finishes of the pads before and after the mechanical load.

values of the measured parameters have been different. It has been very surprising that exposition of $\mathrm{Cu}$ surface of the pads in vapors of $\mathrm{H}_{2} \mathrm{SO}_{3}$ has not caused significant decrease of quality of the joints. Quality of the joints fabricated on the pads exposed in $100 \%$ R.H. is also comparable with the quality of the joints fabricated on clean pads. The low quality of the joints on the pads with Ni finish is a little bit surprising. $\mathrm{Ni}$ is sometimes used also as filler and therefore we have assumed better quality of the joints on the pads of this type. On the other hand low quality of the joints on the pads with Sn finish (HAL) has been assumed [5].

The reason, why the quality of joints on the surfaces of the pads, which have been exposed at high humidity or vapors of $\mathrm{H}_{2} \mathrm{SO}_{3}$ is, according to our opinion, contraction of the adhesive during curing. This contraction will cause movement of particles of filler on the surface of the pad and this movement will 
break the film of oxide or salt of an acid. However, it is assumed that reliability of such the joints, especially the joints fabricated on the surfaces covered with salt of an acid, will be substantially lower in comparison with reliability of the joints fabricated on clean $\mathrm{Cu}$ surface, especially, if the joint will be exposed at higher humidity.

\section{Conclusions}

Quality of the surfaces of the pads intended for assembly of components by the use of electrically conductive adhesives can influence quality of the joints substantially. It is obvious that the components will not be mounted on the pads exposed in vapors of $\mathrm{H}_{2} \mathrm{SO}_{3}$, on the other hand, air humidity can cause that the quality of surfaces of pads will be comparable with the quality of pads especially prepared for our experiments. It has been found that the investigated electrical parameters of the joints fabricated on clear pads are not too different in comparison with the electrical parameters of the joints fabricated on the pads exposed in humidity and in vapors of the acid $\mathrm{H}_{2} \mathrm{SO}_{3}$.

This work has shown that the quality of the adhesive joints is more influenced by the material of the pad than by the cleanness of its surface.

\section{ACKNOWLEDGMENT}

The work has been supported by a research project No. MSM6840770021 (34/13113/05212) "Diagnostics of materials" and by a research project of the Grant Agency of the Czech Rep. No. 102/04/0590 "Development of microelectronics packaging technologies for 3D circuits and systems"

\section{REFERENCES}

[1] J. Liu, Conductive adhesives for electronics packaging, Electrochemical Publications, Chapt. 3, 2005, ISBN 0 90115037

[2] M. A. Gaynes et al., Evaluation of contact resistance for isotropically conductive adhesives, IEEE Trans. CPMT B, Advanc. Pack., vol 18, No. 2, pp. 299-304, 1999

[3] Kim, Jong-Min, Isotropic Conductive Adhesives with Fusible Filler Particles, Journal of Electronic Materials,

http://www.findarticles.com/p/articles/mi_qa3776/is_2 00411/ai_n9462676/pg_2, 2004

[4] P. Mach et al., Diagnostics of adhesive bonds, Proc. 3rd Europ. Microel. and Pack. Symp., IMAPS Europe, Prague, Czech Rep., pp. 83-88, 2004

[5] J. Liu, Surface characteristics, reliability and failure mechanisms of tin, copper and gold metalizations, Proc. Adhesive Electron. 96, Stockholm, Sweden, pp. 141-153, 1996 